In all the above cases the animals survived for at least eighteen months after the beginning of the experiments without developing any symptom of scrapie.

The whole of the experiments therefore had negative results.

It will be noticed that some of the sheep figure under different headings, as different methods of attempting to infect them were employed.

None of the rams used in connection with the experiments became infected.

Natural Transmission from Diseased Ewes to their Lambs.

Out of eleven lambs which were borne by infected ewes and which sucked their dams until they were over four months old only two developed the disease.

\title{
A NOTE ON PIROPLASMOSIS OF THE DONKEY.
}

By J. A. GRIfFiths, Veterinary Officer, Nyasaland.

Piroplasmosis has been recorded as occurring in the donkey by observers in various parts of Africa. In German East Africa it was recorded by Schellhase ${ }^{1}$ in I9I4.

My observations of the disease have been made in the North Nyasa district of Nyasaland and in the New Langenburg and Iringa districts of German East Africa.

The disease appears to affect more severely the white Zanzibar donkey and crosses of this breed with the native grey donkey, while the latter breed has a high degree of natural immunity. This immunity, or at least greater power of resistance, which the grey donkey has, is probably due to the breed being more hardy in every way, and better able to withstand the rough conditions of both climate and food.

In its most common form the disease runs a chronic course, and parasites are not usually plentiful in smears of the peripheral blood.

The parasites vary considerably in shape-amoboid, round, ring, and match-shaped forms being noted at different periods during the course of an acute attack, in the order mentioned. Pear-shaped parasites were rare. Occasionally parasites were seen in pairs, these being either leaf-shaped or match-like forms, and in several cases, where the parasites were joined at the extremities away from the nucleus, a small granule of chromatin was seen at the point of junction.

The nucleus, which is usually towards the periphery in the amoboid, round, and ring forms, is in these cases much smaller than in the pear-shaped and match-like varieties, where it consists of a darkly stained, irregularly rounded mass at one end of the parasite.

1 Extract in "Tropical Veterinary Bulletin," June 1914, No. 2. 
In all the forms above-mentioned the cytoplasm has a reticulate structure. No cross forms have been seen in any of the cases so far examined.

Free parasites have been seen in the plasma during the course of acute infections, these being amoboid and round forms.

The number of parasites in the blood varies daily, and often, even during the course of an acute attack, they may be absent.

The highest infection noted was 4 per cent.; that is, however, exceptionally high, I per cent. being a fairly heavy infection, if one may judge by the gravity of the clinical symptoms. In the chronic cases the parasites, even when present, are often difficult to find, there being perhaps not half a dozen in the whole smear.

The smaller round forms of the parasite have the appearance of cocci, it being difficult to distinguish the nucleus from the cytoplasm. These forms are almost invariably placed towards the periphery of the corpuscle.

In several cases the match-shaped forms with the darkly stained, rounded nucleus at one end were seen in various stages of longitudinal division, there being a portion of the divided nucleus in each segment. A common variation of the match-like form is a parasite whose cytoplasm is sprayed out in a fan-like manner from the nucleus. A few small rod-like curved forms that have been observed applied to the periphery of the corpuscle also appear to be a variation of the match-like form.

In one lot of eleven animals, brood mares and foals, eight were found to be suffering from the disease and had parasites in the peripheral blood. All these animals were emaciated, and one of them died.

Clinically the infected animals show, in the chronic form of the disease, the symptoms of anæmia. The animal is dull and loses all interest in its surroundings, the mucous membranes are pale, and there is gradual emaciation with progressive weakness. There is no febrile reaction. A temperature in this form indicates some secondary infection by other organisms.

During an acute attack the mucous membranes may be at first slightly icteric, later petechiæ become visible, and there is always a rise of temperature to $104^{\circ}$ or $105^{\circ} \mathrm{F}$., with a quickened pulse. The common complication in these cases is broncho-pneumonia, but others, such as pyæmia and septicæmia, have been observed following minor injuries. Mares in foal may abort, and where the disease is secondary to cylicostome infection a fairly high mortality is to be expected. In one lot of eighty animals, a miscellaneous collection of brood mares and their offspring up to two and three year olds, in a low-lying district on the shores of Lake Nyasa, where the greater part of the grazing area was flooded or marshy for six months in the year, piroplasmosis occurred with heavy infection by cylicostomes, and during an observed period of two years 25 per cent. of the animals died.

The period of convalescence is always very prolonged in cases that have once become emaciated, and a great deal of attention to dieting and general hygiene is necessary if the animal is ever likely to regain its former health and condition. Many animals recover but always remain in poor condition, without one being 
able to detect any signs of ill-health, apart from the latter. In such cases parasites may be present in the blood, but not constantly, and when they are, so few that they are not easily found.

In treating the condition trypanblue, a 2 per cent. solution in normal saline, has been given intravenously in doses up to $60 \mathrm{cc}$. without any apparent beneficial result. The effect of animals being well fed is to give the power to recover naturally. Iron preparations and nux vomica are useful in the chronic cases, but only as an aid to a liberal diet.

\section{gevient.}

Veterinary Post-mortem Technic. By Walter J. Crocker, B.S.A., V.M.D. Professor of Veterinary Pathology, School of Veterinary Medicine University of Pennsylvania. With 142 illustrations. Philadelphia and London: J. B. Lippincott Company, r9i 7.

Professor Crocker in the introduction to this text-book expresses the opinion that "practitioners should, whenever possible, autopsy every case lost." Probably every practitioner will agree, but very few conform to the counsel. What is more to the point in the present connection, very many practitioners do not know how to make a proper post-mortem examination, and that is largely due to the inadequate instruction which they received as students.

The first important point with regard to post-mortem examination is that the operator must work according to a definite plan, which prescribes the order in which the different parts of the body are to be examined and the method by which they are in turn to be exposed. The guiding consideration in forming such a plan is that the procedure employed to expose any organ or part must not involve the destruction or mutilation of parts not yet examined.

Professor Crocker's work is intended to guide the student to such an orderly or systematic procedure in conducting post-mortem examinations on animals of the different domesticated species.

The busy practitioner would probably find fault with it on the ground that it has in view post.mortem examinations which only an expert pathologist can be expected to carry out, but the author has rightly decided to treat the subject systematically and fully.

There is, of course, room for difference of opinion with regard to the order in which the different parts of the body should be examined and the best method of exposing them, and it is probable that a good many pathologists will dissent from the author's view that the best position of the carcase for exposing the abdominal organs of the horse is on the left side. Upon the whole, however, the work may be commended as one which will lighten the labour of those who have to teach pathology, and help students to acquire the habit of making post-mortem examinations in a scientific manner. The illustrations, although somewhat crude from an artistic point of view, are very serviceable as a supplement to the instructions regarding cutting operations given in the text. 